\title{
Supporting Information: Tracking Bulk and Interfacial Diffusion using Multiplex Coherent Anti-Stokes Raman Scattering Correlation Spectroscopy
}

\author{
Karen A. Bailey and Zachary D. Schultz* \\ Department of Chemistry and Biochemistry, University of Notre Dame, Notre Dame, IN 46556, \\ USA \\ *Email of corresponding author: Schultz.41@nd.edu
}

\begin{abstract}
:
This supplement contains additional experimental details and results obtained from the coherent anti-Stokes Raman scattering correlation spectroscopy (CARS-CS) measurements. Figure S-1 illustrates how the optical resolution was determined by imaging polymer beads. Table S-1 summarizes the focal radius, axial length, and effective focal volume for each objective used in our experiments to excite the sample for CARS-CS measurements. Figure S-2 is a sample diagram of the position of the excitation volume focused at the air/solution interface, glass/solution interface, and in the bulk medium. Table S-2 lists the mean diffusion coefficients for $300 \mathrm{~nm}$ and $1.10 \mu \mathrm{m}$ polystyrene beads at the two interfaces and in bulk. Dynamic light scattering measurements ascertained the average size of multilamellar vesicles, in Figure S-3, used in CARS-CS experiments.
\end{abstract}




\section{Experimental}

In order to analyze the autocorrelation result, the lateral resolution of our CARS microscope was determined by imaging $300 \mathrm{~nm}$ diameter polystyrene beads (PSB) dried on a glass slide. Figure S-1A and S-1B show good agreement between the brightfield image of an isolated PSB and the CARS image using a 0.9 NA air objective. The CARS spectrum on and off the polystyrene bead demonstrates the chemical specificity, such that signal from the $2850 \mathrm{~cm}^{-1}$ and $2930 \mathrm{~cm}^{-1}$ vibrational bands, indicative of the aliphatic $\mathrm{CH}_{2}$ and $\mathrm{CH}_{3}$ stretches, are only present when the particle is in the excitation volume. The effective focal radius was determined by plotting the intensity profile of the bead and fitting the full width at half maximum (FWHM) in Figure S-1C. The optical resolution for each objective is summarized in Table S-1, which are the coefficients, $r_{0}, z_{0}$, and $V_{\text {eff }}$, used in the autocorrelation fit for each corresponding kinetic series.

We employed multiplex CARS-CS to monitor diffusion dynamics of $300 \mathrm{~nm}$ and 1.10 $\mu \mathrm{m}$ PSB samples at two different interfaces and in the bulk medium illustrated in the sample diagram in Figure S-2. The mean diffusion coefficients for $300 \mathrm{~nm}$ and $1.10 \mu \mathrm{m}$ polystyrene beads at the air/solution interface, glass/solution interface and in the bulk medium are listed in Table S-2.

Multilamellar vesicles (MLV) of 1,2-dipalmitoyl-sn-glycero-3-phosphocholine (DPPC) and 1,2-dioleoyl-sn-glycero-3-phosphocholine (DOPC) were independently sized using dynamic light scattering. Depicted in Figure S-3, the mean size of DOPC vesicles is $1.61 \pm 1.35 \mu \mathrm{m}$ in diameter. In comparison, DPPC is a polydisperse lipid solution with averages of $1.67 \pm 0.84 \mu \mathrm{m}$ and $3.94 \pm 2.48 \mu \mathrm{m}$ diameter vesicles. 
Table S-1. Summary of lateral and axial resolution for each objective.

\begin{tabular}{c|c|c|c}
$\begin{array}{c}\text { Objectiv } \\
\mathrm{e}\end{array}$ & Air $_{1}{ }^{*}$ & Air $_{2}$ & Oil Immersion \\
\hline $\mathrm{NA}$ & 0.65 & 0.9 & 1.3 \\
\hline $\mathrm{r}_{0}(\mathrm{~nm})$ & 675 & $223 \pm 13$ & $173 \pm 30$ \\
\hline $\mathrm{z}_{0}(\mu \mathrm{m})$ & 1.19 & $2.15 \pm 0.19$ & $1.95 \pm 0.16$ \\
\hline $\mathrm{V}_{\text {eff }}(\mathrm{fL})$ & 2.989 & 0.210 & 0.115
\end{tabular}

*The resolution for this objective was based on theoretical calculations for the diffraction limit, $\mathrm{r}_{0}=\frac{0.61 \lambda_{\mathrm{P}}}{\mathrm{NA}}$, and coherence length, $\mathrm{z}_{0}=\frac{2 \lambda_{\mathrm{S}} \lambda_{\mathrm{P}}}{2 \lambda_{\mathrm{S}}-\lambda_{\mathrm{P}}}$.

Table S-2. Mean diffusion coefficients for $300 \mathrm{~nm}$ and $1.10 \mu \mathrm{m}$ diameter PSB observed at different interfaces and in bulk.

\begin{tabular}{c|c|c}
$\begin{array}{c}\text { Sample } \\
\text { Configuration }\end{array}$ & $\begin{array}{c}\text { Mean Diffusion Coefficient }\left(\mathrm{cm}^{2} / \mathrm{s}\right) \\
\text { For } 300 \mathrm{~nm} \text { PSB }\end{array}$ & $\begin{array}{c}\text { Mean Diffusion Coefficient }\left(\mathrm{cm}^{2} / \mathrm{s}\right) \\
\text { For } 1.10 \mu \mathrm{m} \mathrm{PSB}\end{array}$ \\
\hline Air / Solution & $7.28 \pm 1.24 \times 10^{-9}$ & $2.55 \pm 0.50 \times 10^{-9}$ \\
\hline Bulk Solution & $8.63 \pm 0.83 \times 10^{-9}$ & $4.75 \pm 0.57 \times 10^{-9}$ \\
\hline Glass / Solution & $7.98 \pm 1.43 \times 10^{-9}$ & $4.23 \pm 0.91 \times 10^{-9}$
\end{tabular}



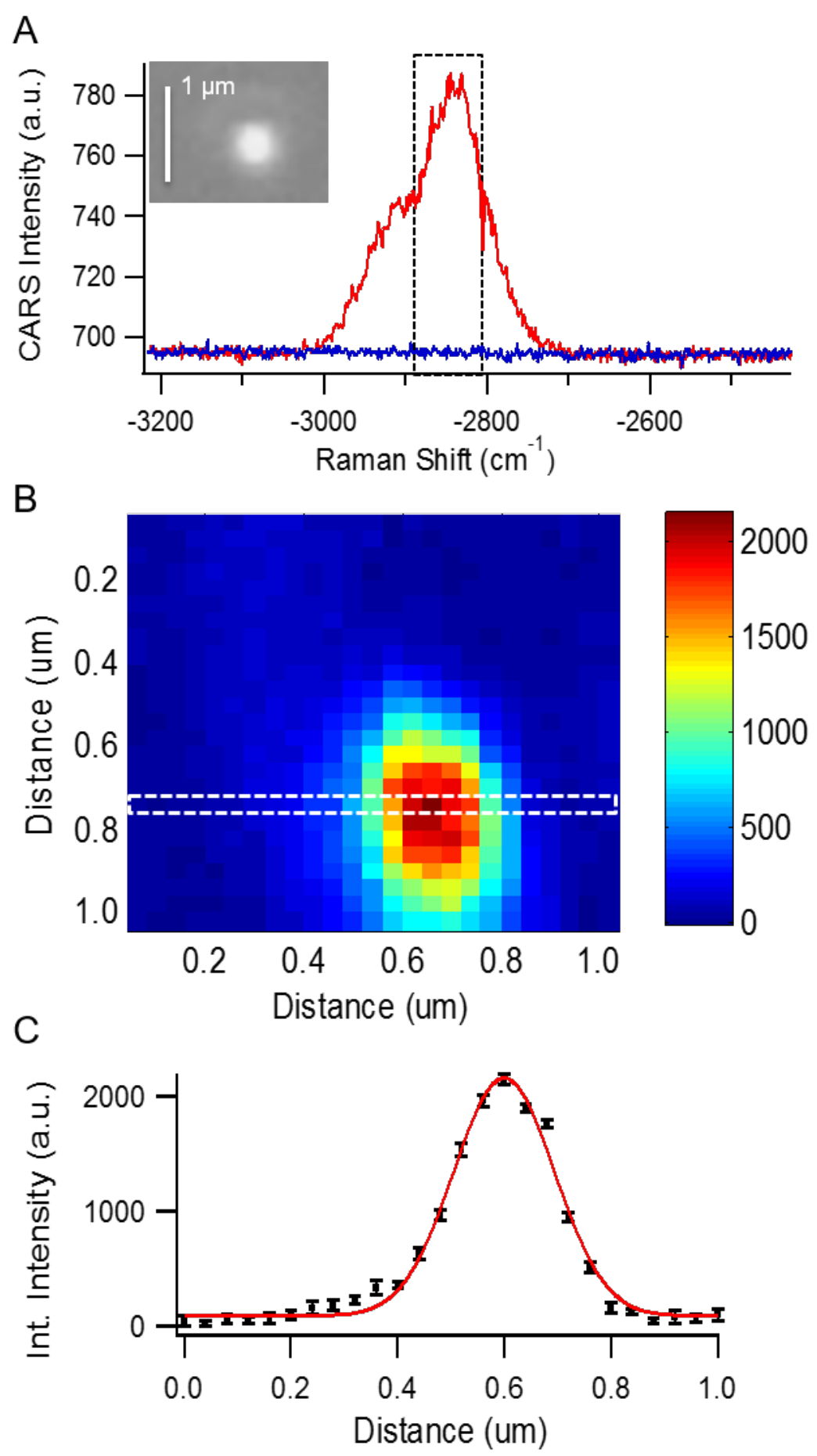

Figure S-1. (A) The multiplex CARS spectrum on (red) and off (blue) a $300 \mathrm{~nm}$ PSB are shown with the brightfield image of the sample (inset). The signal was integrated over the $2850 \mathrm{~cm}^{-}$ ${ }^{1}$ peak to produce (B) the corresponding CARS image. (C) The intensity profile of the highlighted row in (B) is fit to a Gaussian function and used to determine the lateral resolution. Error bars 
represent the standard deviation of the integrated signal. Experimental parameters are: Pump=720 nm, $6.4 \mathrm{~mW}$; Stokes $=909 \mathrm{~nm}, 3.3 \mathrm{~mW}$; Acquisition time $=300 \mathrm{~ms} /$ pixel; Steps $=40$ nm.

A

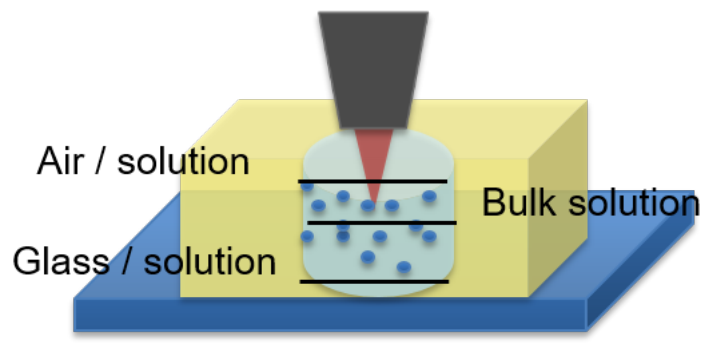

B

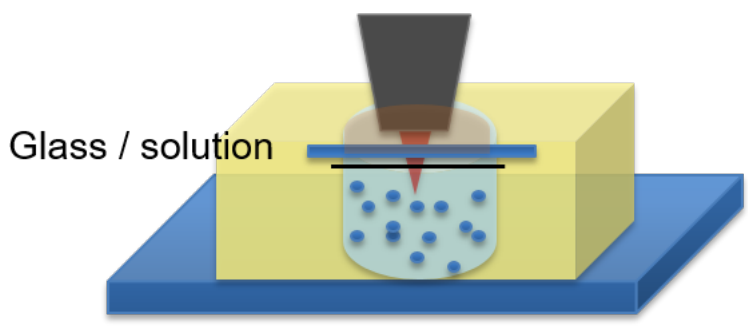

Figure S-2. The sample diagram illustrates the relative position of the excitation volume for measurements at the air/solution and glass/solution interfaces and in bulk for (A) the air objectives and (B) the oil immersion objective.
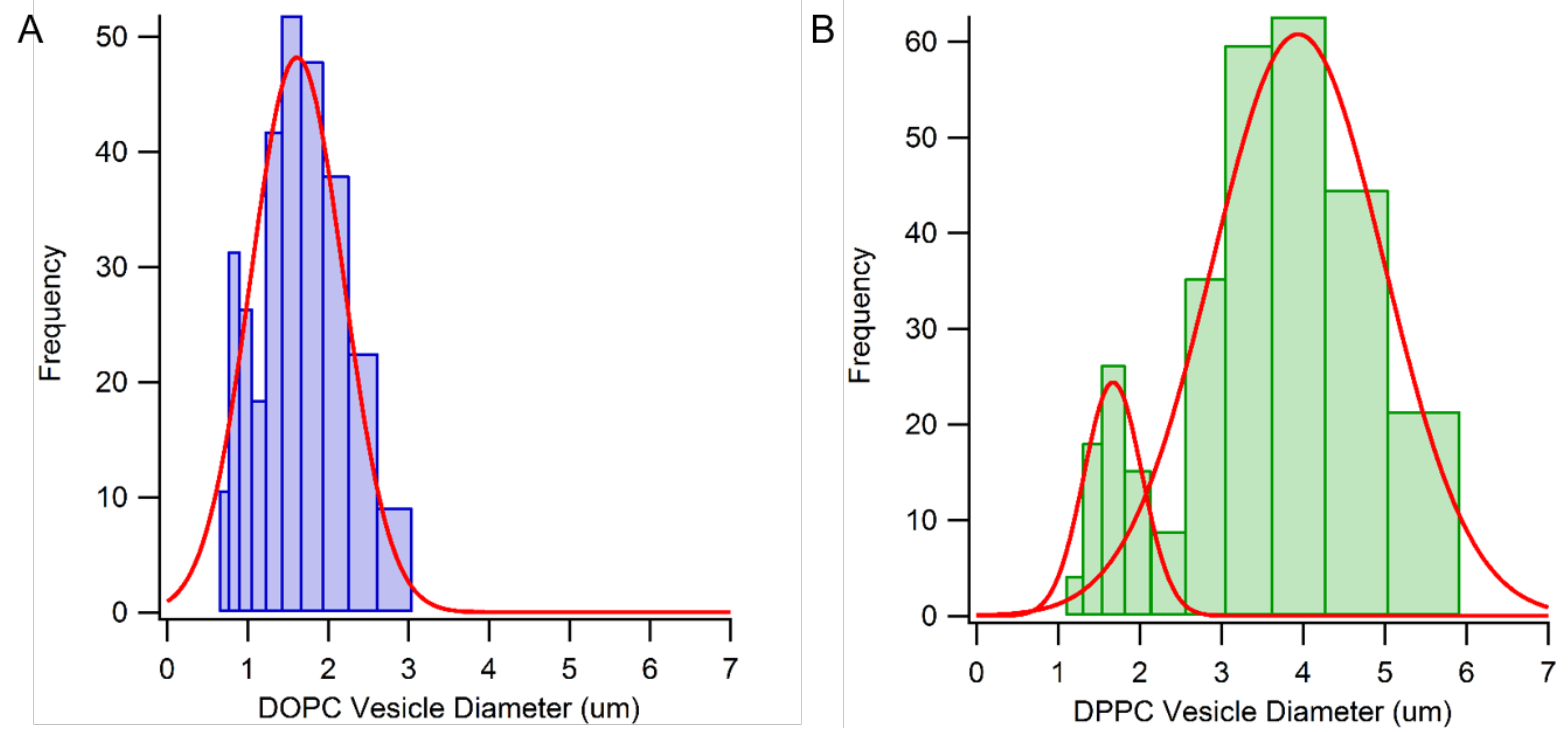

Figure S-3. Dynamic light scattering measurements determined (A) the average size of DOPC is $1.61 \pm 1.35 \mu \mathrm{m}$, whereas (B) DPPC is polydisperse with average sizes of $1.67 \pm 0.84 \mu \mathrm{m}$ and $3.94 \pm 2.48 \mu \mathrm{m}$ in diameter.

\section{References:}


1. J.-X. Cheng, A. Volkmer and X. S. Xie, Journal of the Optical Society of America B, 2002, 19, 1363-1375. 